\title{
Negative Stereotyping Attitudes towards Mental Illness: Is It Cullturally Related?
}

\author{
Tan Kan Ku1, Michael Ha ${ }^{2}$ \\ ${ }^{1}$ Victoria University Institute of Technology (VUIT), St. Albans Campus, McKechine Street, St. Albans P. O. Box \\ 14428, Melbourne, Victoria 8001, Australia \\ ${ }^{2}$ Financial Mathematics Programme, Xi'an Jiaotong-Liverpool University, Suzhou, China \\ Email: tankanku@gmail.com, Tan.Ku@vu.edu.au, Michael.Ha@xjtlu.edu.cn
}

Received 12 November 2015; accepted 11 December 2015; published 18 December 2015

\begin{abstract}
Background: Stigma of mental illness is often related to attitude studies in social science research, cross-cultural psychology and education in social behaviour. Majority of these studies used opinion on mental illness to examine attitudes. Method: A cross-sectional survey was presented to 208 registered nurses in Australia. Principal component analyses (with oblique rotation) were used to identify underlying dimensionality in the correlations of items for negative stereotyping attitudes. Subscale score variations were analysed across nurse type and ethnicity to examine the discriminant validity of the subscales. Results: Principal component analysis (PCA) revealed one dimension accounting for $\mathbf{5 0 . 5 \%}$ of the variations within items for negative stereotyping. Developed as scale, labelled as "Dislike Attributed to Mental Illness (DISL)", reliability analysis indicated high internal consistency with alpha coefficient of .93. Chinese general nurses scored highest on the DISL scale than the other three groups: Chinese psychiatric nurses, Anglo general and Anglo psychiatric nurses. Conclusion: Psychometric evaluation of the Dislike Attributed to Mental Illness (DISL) indicates that it is a reliable scale for measuring negative stereotyping attitudes towards mental illness. The main statistical significance was due to nurse ethnicity.
\end{abstract}

\section{Keywords}

Culture, Stigma, Mental Illness, Negative Stereotyping

\section{Introduction}

A review of international studies on attitudes towards mental illness (for measuring stigma) remained rather negative among health professional, general public members and people with a diagnosed mental disorder. This trend spread over a period of several decades (Ucok et al., 2004 [1]; Angermeyer et al., 2004 [2]; Jorm et al., 2005 [3]; Papadopoulos et al., 2002 [4]; Angermeyer \& Schulze, 2001 [5]).

Stigma related to mental illness is an international concern and a long-standing challenge for research to understand its basis, mechanisms and consequences in order to be able to formulate means by which stigma and its impact may be ameliorated (Arboleda-Florez \& Sartorius, 2008 [6]). Stigma affects patients, contributing to lowering self-esteem (Lv, Wolf \& Wang, 2013 [7]), contributing to the incidence or exacerbation of depression 
(Lin, 1982 [8]), minimizing social inclusion and diminishing social survival by affecting access to employment, housing and other important primary needs, and perhaps, contributing to a cycle of social disadvantage and illness chronicity (Corrigan \& Matthews, 2003 [9]; Lee et al., 2005 [10]). Stigma affects social inclusion of families (Mak \& Cheung, 2012 [11]) and contributes additional burden to their difficult role in care giving (Chang \& Horrocks, 2006 [12]), which subsequently implicated as a significant barrier to informal social network supports which may be important both as a source of relief from psychological distress and a means for gaining access to formal treatment and other relevant formal support.

This paper reports the development of a new approach used in measuring registered nurses' attitudes towards mental illness, the "Dislike Attributed to Mental Illness (DISL)" Scale as part of a Master study which included contact level and cultural values for investigating nurses’ attitudes (Ku, 2007 [13]; Ku \& Ha, 2015 [14]; Ku, Ha \& Siriwan, 2015 [15].

\section{Methods}

\subsection{Subjects}

The sample comprised of 208 nurses (49 Chinese-Australian and 83 Anglo-Australian psychiatric nurses, and 35 Chinese-Australian and 41 Anglo-Australian general nurses). One hundred and forty eight (148) were females and 60 were males. The mean age of the sample was 44.8 years (s.d. $=9.6$ ), ranging from 21 to 65 years of age. For the overall sample the mean number of years working in general setting was 11.2 (s.d. $=12.0$ ) and in psychiatric setting was $9.8($ s.d. $=11.0)$ respectively. Table 1 shows the sample demographics.

As indicated in Table 1, a large number (over 90\%) of psychiatric nurses in the sample had psychiatric training. A smaller number of general nurses than psychiatric nurses, and a smaller number of Chinese General nurses than Anglo nurses, had psychiatric training. This difference was statistically significant. Though the majority of nurses underwent nursing training through a course of study, ranging between $80 \%$ to $100 \%$, when comparing with the sources of training, there were no significant differences in relation to training course, inservice training or other forms of experience.

\subsection{Item Development of Dislike Attributed to Mental Illness (DISL) Scale-Available from the First Author upon Request}

The DISL contained 16 items based on the first author's experience as a psychiatric nurse. This new approach examined the nurses' personal attitudes towards undesirable personal attributes and behaviours in general, and later in the questionnaire, the extent to which these attributes were ascribed by the respondent to people with a

Table 1. Characteristics of the sample $(\mathrm{N}=208)$.

\begin{tabular}{|c|c|c|c|c|c|}
\hline & $\begin{array}{l}\text { Chinese } \\
\text { Psychiatry } \\
(\mathrm{n}=49)\end{array}$ & $\begin{array}{l}\text { Chinese } \\
\text { General } \\
(\mathrm{n}=35)\end{array}$ & $\begin{array}{l}\text { Anglo } \\
\text { Psychiatry } \\
(n=83)\end{array}$ & $\begin{array}{l}\text { Anglo } \\
\text { General } \\
(n=41)\end{array}$ & $\chi^{2 / F}$ value \\
\hline $\mathbf{A g e}^{\#}($ mean, s.d. $)$ & $50.3(5.8)$ & $47.9(6.0)$ & $43.1(9.6)$ & $39.5(11.6)$ & $13.2^{* * *} \mathrm{~F}(1,197)$ \\
\hline Sex (male/female) & $25 / 24$ & $1 / 34$ & $31 / 52$ & $3 / 38$ & $35.4^{* * * *}$ \\
\hline Psy. training(\%) & 95.9 & 28.6 & 90.4 & 61.0 & $67.3 * * *$ \\
\hline Course & 80.9 & 90.0 & 84.0 & 100 & $5.5 \mathrm{~ns}$ \\
\hline In-service & 6.4 & 0 & 12.0 & 0 & $5.0 \mathrm{~ns}$ \\
\hline Others & 12.8 & 10.0 & 6.7 & 0 & $3.9 \mathrm{~ns}$ \\
\hline \multicolumn{5}{|c|}{ Years in Mental Health Nursing (mean, s.d.) } & $\mathrm{F}(1,200)$ \\
\hline & $19.5(10.4)$ & $0.03(0.1)$ & $12.9(9.2)$ & $0.07(0.2)$ & $68.8 * * *$ \\
\hline \multicolumn{5}{|c|}{ Years in General Nursing (mean, s.d.) } & $\mathrm{F}(1,204)$ \\
\hline & $7.7(10.5)$ & $23.1(9.0)$ & $6.1(9.3)$ & $15.6(12.5)$ & $27.3 * * *$ \\
\hline
\end{tabular}

${ }^{\#} 7$ missing cases are excluded from the analysis in age. ${ }^{* * *} p<0.001$. For all $\chi^{2}$ analyses, $\mathrm{df}=3$. 
“mental illness”. Each section contained 16 attributes such as "aggressiveness”, "incoherence”, "unmotivated”, “apathy”, “manipulative”, "hostile”, “avoidant”, “suspicious”, “insensitive”, “uncooperative”, “suspiciousness”, “apathy”, "unmotivated”, submissiveness”, “avoiding”, “incoherence” and "impulsiveness”. These items were conceived as part of a means of measuring stigma (viz, negative stereotyping) towards patients with a mental illness. There were four "dislike" responses for each item. A score of "1" represented "not at all”, "2" represented dislike “a little”, “3” represented “dislike much” and “4” represented “dislike very much” for a particular behaviour or attribute.

For the attribution score (to people with mental illness) each question was responded on the following response scale: " 1 ” for "not at all”, " 2 " for "a little”, “ 3 ” for "much” and " 4 ” for "very much" to the question of “To what extent do you think the following attributes describe with people with severe mental disorders?”. The product of level of dislike and level of attribution to mentally ill patients was averaged over all items to represent the "stigma” (DISL) score, referred to as negative stereotyping.

\subsection{Procedure}

Participants were recruited through a snowballing technique. After the University of Melbourne Human Research Ethics Committee (HERC No. 020030) approved the study, an initial pool of general and psychiatric nurses $(n=20)$ of Chinese-Australian and Anglo-Australian backgrounds working in various institutions were identified and asked to participate in the study. Nurses in the initial pool known to the first author (Ku) were asked to approach potential participants and ask for permission to approach them to introduce the study formally. Nurses who expressed an interest in participating were asked to meet with Ku for the purpose of further explanation of the nature, purpose and procedure of the study. All participants signed a written consent form to anonymous participation. Data collection was achieved in the latter part of 2002 and early 2003.

\subsection{Response Rate}

Three hundred and forty-seven (347) surveys were disturbed. Two hundred and eight nurses out of 331 relevant participants returned the survey giving a $63 \%$ response rate $(208 / 331 \times 100)$.

\subsection{Statistical Analysis}

Principal component analyses were used to identify common dimensions underlying the variation of the item scores of the DISL. Cronbach's alpha coefficients were calculated to estimate the internal reliability of the derived DISL subscales. Two-way analysis of variance was used to examine the discriminant validity of the subscales. All analyses were conducted using the Statistical Package for the Social Sciences (SPSS Version 12).

\section{Results}

\subsection{Dimensions of the Dislike of Negative Behaviour (DISL)}

The new approach developed in the study to measure attitudes towards the mentally ill, which proposes a score based on the product of negatively regarded personal attributes (in general) and the extent to which these are attributed to those with a mental illness.

Proportions of the sample reporting dislike for the attributes appear on the left of Table 2 and proportions of the sample ascribing the attributes to patients with mental illness on the right. For example, aggressiveness was disliked in people in general by the overwhelming majority of the sample (93\%) and nearly 60 percent of nurses ascribed this as typical of a person with mental illness. The most generally disliked personal attributes (with higher than $50 \%$ of the sample disliking them) were: aggressiveness, manipulative, hostility, controlling, insensitivity, unreliability, demanding, attention seeking, and uncooperativeness. The frequency responses of ascribing these characteristics to the mentally ill (50\% and above) indicated that the mentally ill were regarded as suspicious, unmotivated, aggressive, impulsive, unreliable, hostile, incoherent, impulsive, avoiding, uncooperative, manipulative and apathetic.

A Principal Component Analysis (PCA) was conducted on the dislike scale items (that is, product scores which weight one's ascription of a characteristic relating to mentally ill patients by one's level of general dislike of that characteristic) to identify possible subscales. Using the Kaiser's criterion, three components were extracted with eigenvalues higher than or equal to one. However, the first factor accounted for 50.5\% of the va- 
Table 2. Dislike attributed to mental illness scale for measuring negative stereotyping.

\begin{tabular}{llll}
\hline Item & $\begin{array}{l}\text { Personal Dislike of Characteristics } \\
\text { Frequency }(\%)^{1}\end{array}$ & $\begin{array}{l}\text { Characteristics Ascribed to Mental Illness } \\
\text { Frequency }(\%)^{2}\end{array}$ \\
& & & \\
\hline Aggressiveness & $172(92.7)$ & Suspiciousness & $139(67.8)$ \\
Manipulative & $166(79.8)$ & Unmotivated & $136(66.7)$ \\
Hostility & $160(76.9)$ & Aggressiveness & $122(59.5)$ \\
Controlling & $150(72.5)$ & Unreliability & $121(59.0)$ \\
Insensitivity & $137(65.9)$ & Hostility & $118(57.5)$ \\
Unreliability & $133(64.0)$ & Incoherence & $116(56.9)$ \\
Demanding & $133(63.9)$ & Avoiding & $114(55.6)$ \\
Attention seeking & $125(60.0)$ & Impulsiveness & $112(54.6)$ \\
Uncooperativeness & $113(54.3)$ & Insensitivity & $111(54.4)$ \\
Suspiciousness & $90(43.2)$ & Demanding & $107(52.7)$ \\
Apathy & $75(36.2)$ & Uncooperativeness & $106(52.0)$ \\
Unmotivated & $72(35.1)$ & Manipulative & $106(51.9)$ \\
Submissiveness & $66(31.9)$ & Apathy & $100(49.0)$ \\
Avoiding & $61(29.3)$ & Attention seeking & $88(43.3)$ \\
Incoherence & $60(29.9)$ & Controlling & $77(37.7)$ \\
Impulsiveness & $40(19.2)$ & Submissiveness & $55(27.1)$ \\
& & &
\end{tabular}

${ }^{1}$ Sum of ordinal scale, much and very much $(3+4)$ of questionnaire item C; ${ }^{2}$ Sum of ordinal scale, much and very much $(3+4)$ of questionnaire item F.

riance in the items suggesting that possibly one dimension might be sufficient to describe a general negative attitude towards those with mental illness. Also, higher dimensional solutions did not suggest any interpretable content themes for the various factors. Table 3 shows the item loadings of the one factor extraction. Most items, with the exception of submissiveness and impulsiveness had at least $40 \%$ of their variance explained by this component. Treating the items as a scale, reliability analysis revealed very high internal consistency among the items with an alpha coefficient of 0.93, as indicated in Table 3.

\subsubsection{Discriminant Validity}

A two-way analysis of variance was conducted to explore the differences in DISL scores between ethnic groups and nurse types. The main effect of ethnicity was statistically significant $(\mathrm{F}(1,201)=4.85, p<0.05)$. Inspection of the means indicated that Chinese nurses scored higher on this scale (mean $=7.41$, s.d. $=2.56$ ) than Anglo nurses $($ mean $=6.60$, s.d. $=2.41)$. The main effect for nurse type $(\mathrm{F}(1,201)=1.01, p>0.05)$ and the interaction term $(\mathrm{F}(1,201)=0.05, p>0.05)$ were not significant. It is suggested in Figure 1 that Chinese general nurses scored highest in this "stigma" scale (mean $=7.67$, s.d. $=2.87$ ) than the other three groups of nurses: Chinese psychiatric nurses (mean = 7.22, s.d. $=2.33$ ), Anglo general nurses (mean = 6.79, s.d. $=2.24)$, and Anglo psychiatric nurses $($ mean $=6.51$, s.d. $=2.50)$, but the main statistically reliable difference was only due to nurse ethnicity.

\subsubsection{Association between Stigma and 0ther Factors}

Correlations between al stigma and other measures (background demographics) are summarized in Table 4. These are direct correlations without taking account possible confounding between variables. Nevertheless it is instructive to examine these with the view to further "unpacking” of the relationships among variables. Evidently as shown in Table 4, there is no correlation between negative stereotyping attitudes with contact level or background factors.

\subsubsection{Relationship between Group Membership and DISL Scores}

In regard to DISL scores, as indicated in the Simple Model (shown in Table 5), there was only a significant main effect of ethnicity with Chinese nurses (mean =7.41, s.d. $=2.56$ ) endorsing higher dislike attributes (negative stereotyping attitudes) than Anglo nurses (mean=6.60, s.d. = 2.41) towards mental illness.

Analysis of covariance was conducted to examine differences in DISL scores between nurse type and ethnic 
Expressed Dislike by Nurses

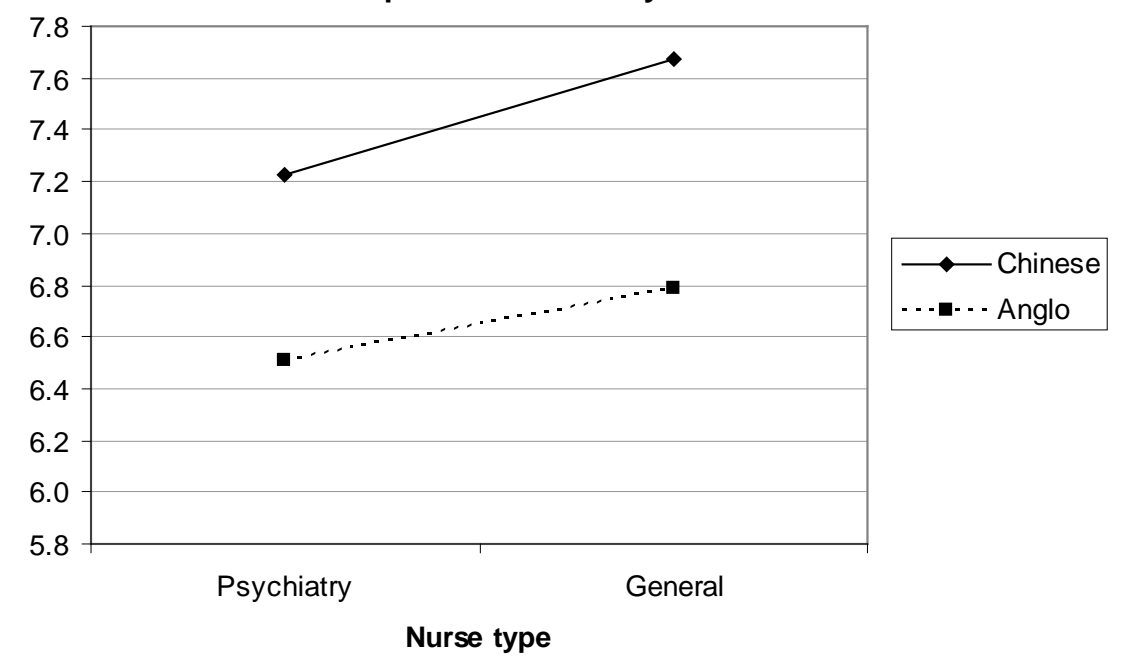

Figure 1. Negative stereotyping attitudes by Nurses.

Table 3. Component matrix for the DISL scale.

\begin{tabular}{lcc}
\hline Item & Component 1 & Communality \\
\hline Avoiding & .79 & .62 \\
Demanding & .78 & .61 \\
Hostility & .78 & .60 \\
Controlling & .77 & .59 \\
Uncooperativeness & .76 & .58 \\
Manipulative & .76 & .57 \\
Attention seeking & .75 & .56 \\
Suspiciousness & .75 & .56 \\
Unmotivated & .70 & .50 \\
Aggressiveness & .70 & .49 \\
Apathy & .69 & .48 \\
Insensitivity & .69 & .47 \\
Incoherence & .68 & .46 \\
Unreliability & .66 & .44 \\
Submissiveness & .60 & .36 \\
Impulsiveness & .45 & .20 \\
\hline Percent variance & & Total variance $=50.5$ \\
Cronbach's alpha & .93 & \\
\hline
\end{tabular}

Table 4. Pearson correlations between stigma (DISL Score) and background measures $(\mathrm{N}=28)$.

\begin{tabular}{lc}
\hline & $\begin{array}{c}\text { Negative Stereotyping } \\
\text { (DISL Score) }\end{array}$ \\
\hline Background Factors & \\
AGE & $-.04 \mathrm{~ns}$ \\
SEX & $.12 \mathrm{~ns}$ \\
YR_MH_NU & $-.04 \mathrm{~ns}$ \\
YR_GE_NU & $.09 \mathrm{~ns}$ \\
\hline ContactFactors & \\
CWS & $-.08 \mathrm{~ns}$ \\
PHN & $-.07 \mathrm{~ns}$ \\
RMI & $.04 \mathrm{~ns}$ \\
ESP & $-1.2 \mathrm{~ns}$ \\
\hline
\end{tabular}

${ }^{*} p<0.05,{ }^{* * *} p<0.001$; ns, not significant. YR_MH_NU = Years in Mental Health Nursing; YR_GE_NU = Years in General Nursing; CWS = Contact Through Work Situation; PHN = Patient Helps Nurse; RMI = Relative with Mental Illness; ESP = External Socialisation with Patient. 
Table 5. Relationship between background variables and DISL scores.

\begin{tabular}{lcll} 
& \multicolumn{3}{c}{ F Values } \\
& Simple & $\begin{array}{l}\text { Model 1 } \\
\text { with background } \\
\text { Fodel }\end{array}$ & $\begin{array}{l}\text { Model 2 } \\
\text { with background } \\
\text { and contact factors }\end{array}$ \\
\hline Nurse type & $1.01 \mathrm{~ns}$ & $.06 \mathrm{~ns}$ & $.07 \mathrm{~ns}$ \\
Ethnic group & $4.85^{*}$ & $4.12 *$ & $4.68^{*}$ \\
Interaction & $.05 \mathrm{~ns}$ & $.04 \mathrm{~ns}$ & $.01 \mathrm{~ns}$
\end{tabular}

${ }^{*} p<0.05$, ns, not significant. ${ }^{1}$ covariates = age, sex, years of mental nursing experience, years of general nursing experience; ${ }^{2}$ covariates $=$ age, sex, years of mental nursing experience, years of general nursing experience, current work environment, and contact levels [(CPP Scale = CWS (via work), PHN (patients helping nurse), RMI (relatives with mental illness), ESP (socializing with person with mental illness)).

groups accounting for the effects of background demographics and contact factors. As shown in Model 1 (Table 5) when background demographics were entered as covariates, the main effect of nurse type was not significant $(\mathrm{F}(1,186)=<1)$. The main effect of ethnicity remained significant $(\mathrm{F}(1,186)=4.12, p<0.05)$. Chinese nurses $($ mean $=7.40$, s.d. $=2.53)$ reported higher dislike attributed to the mentally ill than Anglo nurses (mean $=6.67$, s.d.=2.43). The interaction term was not significant $(\mathrm{F}(1,186)=<1)$ indicating that dislike attributes did not vary across ethnicity and nurse type. There was no simple effect for each individual covariate: age $(F(1,186)=3.73$, $p>0.05)$, sex $(\mathrm{F}(1,186)=2.14, p>0.05)$, years in mental health nursing $(\mathrm{F}(1,186)=1.22, p>0.05)$, and years in general nursing $(\mathrm{F}(1,186)=2.30, p>0.05)$.

In Model 2, when contact type factors were added to demographics as covariates, the main effect of nurse type was not significant $(\mathrm{F}(1,182)=<1)$. The main effect of ethnicity remained as significant $(\mathrm{F}(1,182)=4.68, p$ $<0.05$ ), and not appreciably altered. Examination of the means indicated that Chinese nurses (mean $=7.40$, s.d. $=2.53$ ) again reported higher attributed dislike than Anglo nurses (mean $=6.67$, s.d. $=2.43$ ). The interaction effect was not significant $(\mathrm{F}(1,182)=<1)$. Thus, it would seem that factors other than background demographics and contact level are associated with higher stigma level reported by Chinese nurses as measured by negative attributes ascription to the mentally ill. Again, each demographic and contact factors alone did not account for any effect: age $(\mathrm{F}(1,182)=3.35, p>0.05)$, sex $(\mathrm{F}(1,182)=2.13, p>0.05)$, years in mental health nursing $(\mathrm{F}(1$, $182)=1.21, p>0.05)$, years in general nursing $(\mathrm{F}(1,182)=2.00, p>0.05)$, contact via work $(\mathrm{F}(1,182)=1.08$, $p>0.05)$, patients helping nurse $(\mathrm{F}(1,182)=<1)$, relatives with mental illness $(\mathrm{F}(1,182)=<1)$, and socialising with person with mental illness $(\mathrm{F}(1,182)=1.33, p>0.05)$.

It would appear in general that ethnicity effect on general stigmatising attitudes, measured by the ascription of unfavourable personal attributed, is not entirely accounted for other factors measured in this study, and of importance, not accounted for contact level with mental illness.

\section{Discussion}

Our focus on comparing negative stereotyping attitudes between Anglo and Chinese Australian nurses was guided by suggestion in the literature that stigma of mental illness might be related to cultural variation and contact level with mental illness.

The study by Angermeyer et al., 2004 [2] examined labelling effect on patients with depression and schizophrenia between two countries (Russia and Mongolia). Novosibirsk (Russia) and Ulaanbaatar (Mongolia) were the cities chosen to compare with the previous Germany results by Schulze \& Angermeyer (2003) [16]. The Germany results indicated that patients with depression and schizophrenia anticipated similar level of stigma, however, the latter reported enacted stigmatisation more often than the former. Patients with schizophrenia complained of constant denial access to social roles (partnership and work). These findings were comparable to a study comparing Chinese professionals attitudes towards a case of depression versus schizophrenia in vignette format in the multi-cultural metropolitan city of Melbourne in Australia (Ku \& Ha, 2015) [17].

Having Russians as the majority of its residents, Novosibirsk was an industrial center of Western Siberia, whereas Ulaanbaatar was the largest and capital city of Mongolia where most population adhered to Buddism. Results showed the effects of labelling on public attitudes were comparable between Russia and Mongolia. Labelling as suffering from mental illness correlated positively with endorsement of the need for help, but there was small endorsement of the stereotype of dangerousness. Small differences were noted between the two cities. 
In Novosibirsk, the public tended to express a less desire to help, which was related to a stronger desire for social distance, whereas in Ulaanbaatar, labelling was related to a stronger desire for social distance, consistent with a greater lack of understanding. Greater differences were observed when the data were compared with Germany, where labelling had significant effect on the endorsement of the stereotype of dangerousness, but also evoked the perception of need for help. The authors suggested wider media coverage of associating mentally ill people with violent crime in Germany than in Russia and Mongolia might have affected public attitudes (Angermeyer \& Schulze, 2001 [5]. Angermeyer et al., 2004 [2] contended that labelling effect varied among different cultures and were associated with different components of stigma (social distance, need for help and endorsement of stereotypes of dangerousness) and their findings "support the notion that labelling effects are culturally-related” (p. 420). However, these authors did not venture to explain what aspects of culture were important nor speculate on the relationship between culture and stigma.

A national representative sample in Australia and in Japan were surveyed to obtain opinions in relation to one of four vignettes describing depression, depression with suicidal thoughts, early schizophrenia and chronic schizophrenia (Jorm, et al., 2005) [3]. Results indicated more of the Australian public used psychiatric labels than the Japanese, particularly "depression" when asked about the people portrayed in the vignettes. More of the Australians used the term "schizophrenia" for the early schizophrenia vignette than for the chronic case, whereas the Japanese reserved psychiatric labels only for the more chronic and severe cases of mental disorder. More of the Japanese endorsed "hiding" the mentally ill within the family and more were reluctant to discuss mental illness with others. The Japanese believed in the help of counsellors, and benefits of treatment, but not of general practitioners, and they were less optimistic about full recovery. Australians were positive about the benefits of seeking professional help from counsellors and general practitioners, but negative about psychiatric medications. Hospitalisation and electro-convulsive therapy were negatively regarded in both countries. The authors suggested the differences in beliefs about treatment and recognition of disorders between Australians and Japanese might be explained by the greater openness among the Australian public toward mental disorders and about acceptance of treatments than the Japanese. According to Jorm et al. (2005) [3], Australia emphasises community services whereas Japan emphasises hospital care for the mentally ill, and this was said to influence differences in opinions between the two country samples. Little attempt was made to explain the national differences in view of the well evidenced cultural differences between the two nations (e.g., Hofstede, 2001 [18]).

Jorm et al.'s (2005) [3] findings might be related to endorsement of individualism and collectivism in Australian and Japanese cultures respectively, which correlated with our findings that Anglo-Australian nurses (of individualistic culture) reported less stigmatising attitudes towards mental illness measured by DISL than Chinese-Australian nurses (of collectivistic culture). The DISL Scale captures a wide stereotype relating to the negatively regarded personal attributes ascribed to individuals with mental illness. The measure does not imply any social consequences as to how to behave towards he mentally ill but it does cover personal characteristics that may dictate behaviour in relation to those with mental illness and particularly one's personal aversion for such subjects on the basis of their behaviour. The findings transpired from DISL suggest that Chinese group membership influenced interpretations of the character of the mentally ill person through his behaviour. Results suggest a relationship between culture and negative stereotyping towards the mentally ill, but do not specify why such a relationship occurs.

\section{Conclusion}

The findings generated from DISL indicated that cultural differences (Anglo-Australian versus Chinese-Australian nurses) were not attributable to differences between groups in relation to backgrounds variables or contact with people having a mental illness. Contact was better placed as a mediator in this relationship, particularly among the Chinese group membership (Ku \& Ha, 2015 [14]). Despite such consideration, psychometric evaluation of the DISL suggests it is a reliable scale for measuring one dimension of nurses' attitudes towards patients with a mental illness due to its high internal consistency and enables the study of this relationship in larger samples.

\section{Acknowledgements}

The authors would like to thank Victoria University Institute of Technology and Xian Jiaotong-Liverpool University for supporting and sponsoring the publication of this paper. The first author acknowledges the substantial intellectual contribution of the late Associate Professor Steven Klimidis to the formulation of the study. 


\section{Authors' Contributions}

The first author carried out the study under the supervision of the late Associate Professor Steven Klimidis at The Centre for International Mental Health (CIMH), Department of Psychiatry at The University of Melbourne and co-supervised by Associate Professor Harry Minas, Director at CIMH. The first author prepared the first draft of the paper and the second author verified statistical results and edited the paper. Both authors have approved the final version of the manuscript.

\section{References}

[1] Ucok, A., Polat, A., Sartorus, N., Erkoc, S. and Atakli, C. (2004) Attitudes of Psychiatrists toward Patients with Schizophrenia. Psychiatry and Clinical Neurosciences, 58, 89-91. http://dx.doi.org/10.1111/j.1440-1819.2004.01198.x

[2] Angermeyer, M.C., Buyantugs, L. Kenzine, D.V. and Matschinger, H. (2004) Effects of Labelling on Public Attitudes towards People with Schizophrenia: Are There Cultural Differences? Acta Psychiatrica Scandinavica, 109, 420-425. http://dx.doi.org/10.1111/j.1600-0047.2004.00310.X

[3] Jorm, A.F., Nakane, Y., Christensen, H., Yoshioka, K., Griffiths, K.M. and Wata, Y. (2005) Public Beliefs about Treatment and Outcome of Mental Disorders: A Comparison of Australia and Japan. BMC Medicine, 3, 12. www.biomedcentral.com/1741-7015/3/12

[4] Papadopoulos, C., Leavey, G. and Vincent, C. (2002) Factors Influencing Stigma: A Comparison of Greek-Cyproit and English Attitudes towards Mental Illness in North London. Social Psychiatry and Psychiatric Epidemiology, 37, 430434. http://dx.doi.org/10.1007/s00127-002-0560-9

[5] Angermeyer, M.C. and Schulze, B. (2001) Reinforcing Stereotypes: How the Focus on Forensic Cases in News Reporting May Influence Public Attitudes towards the Mentally Ill. International Journal of Law and Psychiatry, 24, 469-486. http://dx.doi.org/10.1016/S0160-2527(01)00079-6

[6] Arbolea-Florez, J. and Sartorius, N. (2008) Understanding the Stigma of Mental Illness. John Wiley \& Sons Ltd., Chichester. http://dx.doi.org/10.1002/9780470997642

[7] Lv, Y., Wolf, A. and Wang, X. (2013) Experienced Stigma and Self-Stigma in Chinese Patients with Schizophrenia. General Hospital Psychiatry, 35, 83-88. http://dx.doi.org/10.1016/j.genhosppsych.2012.07.007

[8] Lin, T. (1982) Culture and Psychiatry: A Chinese Perspective. Australian and New Zealand Journal of Psychiatry, 16, 235-245. http://dx.doi.org/10.3109/00048678209161263

[9] Corrigan, P.W. and Matthews, A.C. (2003) Stigma and Disclosure: Implications for Coming out of the Closet. Journal of Mental Health, 12, 235-248. http://dx.doi.org/10.1080/0963823031000118221

[10] Lee, S., Lee, M.T.Y., Chiu, M.Y.L. and Kleinman A. (2005) Experience of Social Stigma by People with Schizophrenia in Hong Kong. British Journal of Psychiatry, 186, 153-157. http://dx.doi.org/10.1192/bjp.186.2.153

[11] Mak, W.W. and Cheung, R.Y.M. (2012) Psychological Distress and Subjective Burden of Caregivers of People with mental Illness: The Role of Affiliate Stigma and Face Concern. Community Mental Health Journal, 48, 270-274. http://dx.doi.org/10.1007/s10597-011-9422-9

[12] Chang, K.H. and Horrocks, S. (2005) Lived Experience of Family Caregivers of Mentally Ill Relatives. Journal of Advanced Nursing, 53, 435-443. http://dx.doi.org/10.1111/j.1365-2648.2006.03732.x

[13] Ku, T.K. (2007) Culture and Stigma towards Mental Illness: A Comparison of General and Psychiatric Nurses of Chinese and Anglo-Australian Backgrounds. Master Thesis, Department of Psychiatry, The University of Melbourne. http://repository.unimelb.edu.au/10187/8400

[14] Ku, T.K. and Ha, M. (2015) Culture and Stigma of Mental Illness: Path Analysis Conducted with Amos in Transcultural Psychiatry in Australia. Journal of Biosciences and Medicines, 3, 15-22. http://dx.doi.org/10.4236/jbm.2015.36003

[15] Ku, T.K., Ha, M. and Siriwan, U. (2015) Professional Attitudes towards Mental Illness: Testing the Contact Hypothesis among Nurses of Ethnic Anglo and Chinese Australian Backgrounds. Open Journal of Social Sciences, 3, 130-138. http://dx.doi.org/10.4236/jss.2015.37022

[16] Schulze, B. and Angermeyer, M.C. (2003) Subjective Experiences of Stigma: A Focus Group Study of Schizophrenic Patients, Their Relatives and Mental Health Professionals. Social Science and Medicine, 56, 299-312. http://dx.doi.org/10.1016/S0277-9536(02)00028-X

[17] Ku, T.K. and Ha, M. (2015) Development of the Attitudes towards Depression and Schizophrenia Scale (ADSS). Studies in Social Sciences and Humanities, 2, 12-20. http://www.rassweb.com

[18] Hofstede, G.H. (2001) Culture’s Consequences: Comparing Values, Behaviours, Institutions, and Organizations across Nations. 2nd Edition, Sage, Thousand Oaks, California. 